\title{
Is there a Change in Clinical Practice in the Imaging of Thoraco-Lumbar Vertebral Injuries? - A Shift in Paradigm to CT Imaging as an Interim Measure
}

\author{
Debkumar Chowdhury* \\ Specialist Registrar in Emergency Medicine, United Kingdom
}

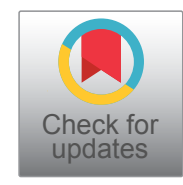

\author{
Keywords \\ Vertebral fractures, Imaging, Computed tomography, Magnetic resonance imaging
}

\section{Commentary}

Plain radiograph imaging of the thoracic and lumbar vertebra still forms the initial modality of choice in the detection of acute bony injury. The wide availability of this modality is possibly the reason for its continued use. In this article imaging of cervical spine injuries will not be discussed as this a topic that requires in depth analysis and is beyond the scope of this article.

The thoracic and lumbar vertebrae can be considered into 3 separate columns- anterior, middle and posterior [1]. Instability occurs when two adjacent columns are affected or when all three columns are affected. There are various classification systems in the management of thoraco-lumbar injuries. For the acute clinician's immediate management, the main concern is instability of the fractures with the need for spinal immobilisation on a spinal bed. The concerns about ligamentous instability would only surface in the intermediate phase once other life-threatening injuries have been excluded.

When considering appropriate imaging of the thoracic and lumbar spine in the context of trauma, unless in the context of the multiply injured patient, is likely to be plain radiograph. In the context of the polytrauma patient CT would include imaging of the vertebrae with MPR. As we know magnetic resonance imaging is the gold standard imaging in the diagnosis of spinal cord injury with specific focus on ligamentous, soft tissue and intervertebral disc prolapse. In the context of trauma leading to cauda equina syndrome (CES) MRI is the gold standard imaging modality of choice. The purpose of this article is not to challenge this but to gain an understanding in the use of CT in defining thoraco-lumbar vertebral fractures.

The main concern with vertebral fractures is unstable fracture fragments at risk of retropulsion causing spinal cord injury with devastating neurological sequelae. In high-speed accidents this can note to be as high as $25 \%$ of patient who suffer spinal cord injury [2]. In the context of acute thoracic vertebral fractures as a result of trauma, there are significant concerns of additional thoracic injuries [3]. The injuries could involve sternal fractures, traumatic cardiac injury and potential injury to lung parenchyma. In the context of thoraco-lumbar fractures, in approximately $25 \%$ of these patients [4] there are other associated spine fractures, hence a meticulous and systematic method of assessment is required.

There is limited availability of MRI imaging when compared to plain imaging and $\mathrm{CT}$ imaging especially in smaller district general hospitals and in the context of out of hours imaging. Even if plain radiograph identifies fractures e.g., chance fractures, avulsion fractures the acute clinician would be concerned about potential unstable fractures based on the mechanism of injuries.

The vast majority of lumbar vertebral fractures are osteoporotic in aetiology and occurs in the context of minor trauma. The question arises of whether these require more definitive imaging with CT or it acceptable that these fractures would be inherently stable thereby not warranting further investigations. Vertebral wedge/compression fractures form upto $50 \%$ of acute lumbar fractures [5], however even in these fractures any associated ligamentous injury need to evaluated through further imaging depending on the clinical situation.

*Corresponding author: Debkumar Chowdhury, Specialist Registrar in Emergency Medicine, United Kingdom

Accepted: August 17, 2021

Published online: August 19, 2021

Citation: Chowdhury D (2021) Is there a Change in Clinical Practice in the Imaging of Thoraco-Lumbar Vertebral Injuries? - A Shift in Paradigm to CT Imaging as an Interim Measure. Sch J Emerg Med Crit Care 5(1):121-122 
The other group of fractures would be at the junction of the thoracic and lumbar vertebrae in the context of motor vehicle accident, particularly between T10-L2 vertebrae [6]. The main reason is due to the relative immobile thoracic vertebra in relation to the more mobile lumbar vertebrae. However, following the systematic implementation of assessing these injuries using ATLS principles has led to improvement in resultant morbidity and mortality [7]. Due to improved sensitivity in detecting acute bony injuries with CT imaging when compared to plain radiographs, some authors have suggested that plain radiographs can be omitted completely [8]. This would add increased workload to the radiology department and the long-term implications of the single dose of radiation is not known.

Potential reasons for the reliance of Computed Tomography as a follow-on imaging from plain radiograph:

$>$ Both acute onset traumatic back pain and acute on chronic back pain is a huge socio-economic burden

Medico-legal considerations

Better able to visualise the vertebral alignment

Imaging as part of multi slice polytrauma imaging

Help better decision making through a multidisciplinary approach

In terms of my own clinical practice, I have noted that there has been more liberal use of CT imaging of the thoracolumbar fractures that would have not been the requested in the absence of focal neurological deficit or significant mechanism of injury. The potential explanation for this shift in the practice could be potentially related to the reasons as stated above. Missed injuries when not done as part of CT polytrauma are significant and with advances in imaging it is no longer for this to acceptable.

There could a potential clinical rationale in choosing CT over plain radiograph as the first modality of choice if multilevel vertebral injuries are clinical suspected. This would remove the need for exposing additional radiation as an additional scan. However, this is not without the problem of high doses of radiation to the thoracic structures when compared to plain radiographs.

As highlighted in this article, with the wider availability of CT imaging more patients would undergo this form of imaging as a primary or follow up modality due to the potential reasons as stated above.

\section{Authors Declaration}

The views expressed in this article are of my own and does not necessarily reflect other clinicians' clinical practice within the current healthcare system.

\section{References}

1. Su Q, Li C, Li Y, et al. (2020) Analysis and improvement of the three-column spinal theory. BMC Musculoskelet Disord 21: 537.

2. Chapman JR, Agel J, Jurkovich GJ, et al. (2008) Thoracolumbar flexion-distraction injuries: Associated morbidity and neurological outcomes. Spine (Phila Pa 1976) 33: 648-657.

3. Singh R, McD Taylor D, D'Souza D, et al. (2009) Injuries significantly associated with thoracic spine fractures: a casecontrol study. Emerg Med Australas 21: 419-423.

4. Wood KB, Li W, Lebl DR, et al. (2014) Management of thoracolumbar spine fractures. Spine J 14: 145-164.

5. Pope TL (2012) Harris \& Harris' Radiology of Emergency Medicine. Lippincott Williams \& Wilkins.

6. Denis $F$ (1983) The three-column spine and its significance in the classification of acute thoracolumbar spinal injuries. Spine (Phila Pa 1976) 8: 817-831.

7. Navarro S, Montmany S, Rebasa P, et al. (2014) Impact of ATLS training on preventable and potentially preventable deaths. World J Surg 38: 2273-2278.

8. Berry GE, Adams S, Harris MB, et al. (2005) Are plain radiographs of the spine necessary during evaluation after blunt trauma? Accuracy of screening torso computed tomography in thoracic/ lumbar spine fracture diagnosis. J Trauma 59: 1410-1413.

DOI: $10.36959 / 592 / 392$

Copyright: (C) 2021 Chowdhury D. This is an open-access article distributed under the terms of the Creative Commons Attribution License, which permits unrestricted use, distribution, and reproduction in any medium, provided the original author and source are credited. 